\title{
As orientações de enfermagem e sua influência nos níveis de ansiedade dos pacientes cirúrgicos hospitalares
}

\author{
Nursing guidance and its influence on surgical hospital patients' anxiety levels \\ Las directrices de la enfermería y su influencia sobre los niveles de ansiedad de los \\ pacientes quirúrgicos de los hospitales
}

\author{
Thays Macedo Nascimento Costa ${ }^{I}$ Carlos Eduardo Peres Sampaio ${ }^{I I}$
}

\begin{abstract}
RESUMO: Estudo descritivo com abordagem quantitativa, visando identificar o traço e estado de ansiedade dos pacientes hospitalares submetidos às cirurgias gerais e descrever a influência das orientações de enfermagem nos níveis de ansiedade do paciente cirúrgico. A pesquisa foi realizada em uma unidade de cirurgia hospitalar, no município do Rio de Janeiro, no período de julho de 2012 a janeiro de 2013, com entrevista estruturada. Participaram do estudo 50 pacientes, cujas idades variaram entre 20-70 anos. Mediante a análise dos resultados, identificou-se que os pacientes que não receberam orientações de enfermagem aumentaram seus níveis de traço e estado de ansiedade. Conclui-se que as orientações de enfermagem no pré-operatório exercem importância fundamental tanto para o cliente como para toda a equipe que participa desse momento, com vistas ao bem-estar e à redução da ansiedade no momento cirúrgico.

Palavra-Chave: Orientação de enfermagem; traço; estado; ansiedade.
\end{abstract}

ABSTRACT: This quantitative, descriptive study to identify trait and state anxiety in hospital patients undergoing general surgery, and describe the influence of nursing guidance on surgical patients' anxiety levels, was carried out using structured interviews at a hospital surgical department in the municipality of Rio de Janeiro, between July 2012 and January 2013,. The study comprised 50 patients, aged from 20 to 70 years. Analysis of the results revealed that levels of trait and state anxiety increased in patients who received no nursing guidance. It was concluded that preoperative nursing guidance is of fundamental importance to both the client and the whole team participating in this event, by fostering well-being and reducing anxiety during surgery. Keyword: Nursing guidance; trait; state; anxiety.

RESUMEN: Estudio descriptivo con abordaje cuantitativo, cuyo objetivo fue identificar niveles de ansiedad de trazo y estado de los pacientes hospitalarios sometidos a las cirugías generales y describir la influencia de las orientaciones de enfermería en los niveles de ansiedad de los pacientes quirúrgicos. La investigación fue llevada a cabo en una unidad de cirugía hospitalaria, en el municipio de Río de Janeiro, en el período comprendido entre julio 2012 y enero 2013, con entrevista estructurada. Participaron del estudio 50 pacientes, con edades variando entre 20 y 70 años. A través del análisis de los resultados, se identificó que los pacientes que no recibieron directrices de enfermería aumentaron sus niveles de ansiedad de trazo y estado. Se concluye que las directrices de enfermería preoperatoria tienen una fundamental importancia tanto para el cliente como para todo el equipo que participa, con vistas al bienestar y a la reducción de la ansiedad en el momento quirúrgico. Palabra Clave: Orientación de enfermería; trazo; estado; ansiedad.

\section{INTRODUÇÃO}

Quando alguma função do organismo não está sendo desempenhada de maneira correta pode se definir como adoecimento ${ }^{1}$. Este processo ocorre quando essas funções estão fragilizadas, e o corpo humano passa a responder de maneira negativa, com a presença de alguns sintomas, como cansaço, cefaleia e aumento da pressão arterial.

O adoecer pode levar à hospitalização e esse evento envolve uma grande capacidade de adaptação do indivíduo às várias mudanças que ocorrem no co- tidiano; o paciente passa a seguir a rotina da unidade agravando as sensações de isolamento e angústia préexistentes, como o medo, a tensão pela internação e a preocupação, o que implica uma série de sentimentos de desconforto. Esse processo proporciona a despersonalização, isto acontece quando o paciente permanece muito tempo internado como, por exemplo, em uma intervenção cirúrgica, passando, portanto, a ser tratado em função do quadro de sintomas que apresenta, e não mais como indivíduo ${ }^{2}$.

'Enfermeira Graduada pelo Curso de Graduação da Faculdade de Enfermagem da Universidade do Estado do Rio de Janeiro. Brasil. E-mail: thaymacedo1505@hotmail.com.

IIEnfermeiro. Doutor. Professor Associado do Departamento de Enfermagem Médico-Cirúrgico da Universidade do Estado do Rio de Janeiro. Orientador da Pesquisa Impacto das orientações de enfermagem no nível de ansiedade dos pacientes cirúrgicos. Rio de Janeiro, Brasil. E-mail: carlosedusampa@ig.com.br. 
O tempo de espera para a realização de um procedimento cirúrgico em pacientes hospitalizados pode ser a causa de grande estresse e angústia. Essas sensações estão diretamente relacionadas tanto à questão invasiva do procedimento, quanto à incerteza do resultado diagnóstico ${ }^{3}$. Nesse contexto de espera e ansiedade, os familiares também estão intimamente envolvidos e compartilham com o paciente seus sentimentos e incertezas, tornando o manejo dessa situação ainda mais complexa para a equipe de enfermagem, visto que esses momentos são presenciados de perto por ela.

Diante dessa problemática, é relevante o acompanhamento dos pacientes cirúrgicos no período pré-operatório pela equipe de enfermagem, visto que é nesse momento que o cliente se encontra a espera da cirurgia e precisa de orientação quanto ao procedimento, aos riscos de possíveis complicações no pós-operatório. Assim, surge a seguinte questão de pesquisa: As orientações de enfermagem exercem influência no nível de ansiedade dos pacientes cirúrgicos hospitalares?

O enfermeiro é de grande relevância, pois presta assistência integral ao paciente cirúrgico, prioriza não apenas os fatores físicos, mais os aspectos psicológicos. Ao iniciar o período pré-operatório com a marcação cirúrgica, o paciente vivencia temores e medos relacionados ao procedimento cirúrgico, logo, é imprescindível o apoio psicoemocional, oferecido pelo enfermeiro, mediante as orientações e cuidados pré-operatórios ${ }^{4}$.

A justificativa pelo interesse desta temática surgiu do Projeto de Iniciação Científica da Universidade do Estado do Rio de Janeiro intitulado: Impacto das orientações de enfermagem no nivel de ansiedade dos pacientes cirúrgicos. A experiência acadêmica em cenários de prática possibilitou observar que grande parte dos pacientes aumenta seu nível de ansiedade devido à espera da cirurgia.

A partir dessa perspectiva, a pesquisa tem como relevância estudar a ansiedade dos pacientes cirúrgicos e os agravos de saúde que os níveis de ansiedade elevados ocasionam no organismo, assim como os aspectos emocionais, fisiológicos e sociais. $\mathrm{O}$ aumento da ansiedade pode ocorrer devido às suspensões cirúrgicas, o que demonstra o seu lado negativo, provocando uma série de prejuízos ao paciente, aumentando seu nível de ansiedade, abalando o seu psicoemocional, o que interfere no resultado da assistência e produtividade dos serviços, implicando custos operacionais e financeiros, sendo prejudicial para a instituição $0^{4}$.

Assim, foi estabelecido como objetivos do estudo: identificar o traço e o estado de ansiedade dos pacientes hospitalares submetidos às cirurgias gerais $\mathrm{e}$ descrever a influência das orientações de enfermagem nos níveis de ansiedade do paciente cirúrgico.

\section{ReVisÃo de LITERATURA}

A assistência de enfermagem pré-operatória é dividida em pré-operatório mediato e imediato. $\mathrm{O}$ pré-operatório mediato compreende o período que vai do momento da marcação da cirurgia até 24 horas antes do procedimento, nesse momento são realizados anamnese, exame físico, nutricional, laboratorial, exames de imagem, preparo físico e emocional e as orientações de enfermagem que objetivam a prevenção de complicações no pós-operatório. Tais orientações incluem: exercícios respiratórios de tosse e respiração profunda; exercícios dos membros inferiores, que previnem a trombose venosa profunda; orienta-se o uso de meia compressiva até a alta hospitalar; e outras orientações que dependem do histórico levantado e dados do exame físico, como tabagismo, obesidade, feridas, alterações em exames complemetares ${ }^{5}$.

Já o pré-operatório imediato, é compreendido desde as vésperas da cirurgia até a chegada do cliente ao centro cirúrgico; o enfermeiro planeja e avalia o estado físico e orienta o paciente a retirar as roupas, a colocar a camisola hospitalar e o gorro de hospital; orienta quanto ao jejum, ao esvaziamento da bexiga, à higiene corporal, capilar e oral, e à remoção de próteses. Essas orientações contribuem para deixar o paciente mais próximo do que será realizado no momento cirúrgico reduzindo os altos níveis de ansiedade 5 .

Além do preparo físico, o enfermeiro tem o papel de trabalhar o emocional do paciente prestando ações de assistência mais diretas a esse paciente no préoperatório. Com isso, passou a utilizar como estratégia a visita pré-operatória de enfermagem, que possibilita perceber o estado de apreensão do paciente e família frente ao momento cirúrgico, esclarecendo possíveis dúvidas e ajudando a detectar problemas. Tem como finalidade diminuir a ansiedade do cliente e de sua família em relação ao procedimento anestésicocirúrgico ${ }^{6}$.

O enfermeiro tem uma visão integral das necessidades humanas básicas do paciente, favorecendo o cuidado tanto do paciente como da família. Para essa assistência, o enfermeiro necessita de um olhar diferencial, de modo holístico, para realizar suas atividades de forma sistematizada, visto que o paciente permanece internado por algum tempo, que o deixa distante dos seus parentes e amigos?

A ansiedade é um fenômeno que ora beneficia, ora prejudica o ser humano, esse sentimento pode estimular o indivíduo a entrar em estado de ação, em excesso faz o contrário, impede reações que paralisam as pessoas, que deixam de fazer coisas simples do dia a dia por causa do desconforto que sentem. Esses acontecimentos podem ser gerados por causa dos transtornos de ansiedade que podem aparecer na forma de preocupações, tensão, medo, sensação de 
que algo ruim vai acontecer e falta de controle sobre o pensamento. Tais reações podem ser mesuradas através da utilização das escalas de estado e traço de ansiedade; essas escalas contribuem para delinear os níveis de ansiedade do paciente cirúrgico ${ }^{8}$.

A escala traço de ansiedade descreve como o indivíduo geralmente se sente. Já, a escala de estado mensura como o indivíduo se sente naquele determinado momento e, também, no passado.

O estado de ansiedade é uma reação emocional transitória, caracterizada por sentimentos de tensão, apreensão, nervosismo, preocupação e inquietação, o que contribui para a alteração da frequência cardíaca, do padrão respiratório, pressão arterial, tremores e sudorese. Já, o traço de ansiedade é o comportamento individual que permanece latente, até que em uma determinada situação, seja por estresse ou ansiedade, são ativados, levando o indivíduo a reagir de uma determinada forma?.

Se o indivíduo interpretar um estímulo interno ou externo como ameaçador, ele desenvolverá o estado de ansiedade, caracterizado com sentimentos de tensão e apreensão, estimulando o sistema nervoso autônomo. Sendo assim, as orientações feitas pelo enfermeiro, nesse momento, são importantes para esclarecer possíveis duvidas e minimizar a ansiedade do paciente ${ }^{10}$.

É importante que o enfermeiro realize as orientações direcionadas ao paciente, tendo o cuidado de responder as perguntas de acordo com o nível de informação e de entendimento do paciente, pois o excesso de detalhes pode confundi-lo aumentar o seu nível de ansiedade. As informações elaboradas pelo enfermeiro devem ser claras e simples para não deixar o paciente confuso e mais ansioso ${ }^{11,12}$.

$\mathrm{O}$ enfermeiro deve estar preparado com informações não apenas técnicas e cientificas, mas de forma humanística e holística, pois muitas vezes as orientações ultrapassam as barreiras técnicas e cientificas, ajudando aquele paciente a encontrar mecanismos de enfrentamentos eficazes para o momento vivenciado ${ }^{11}$.

\section{Metodologia}

Trata-se de um estudo do tipo descritivo, quaseexperimental, com abordagem quantitativa. Essa modalidade de pesquisa tem como objetivo descrever e sintetizar os dados de certo grupo, sem tirar quaisquer conclusões ou interferências sobre o grupo maior ${ }^{13}$.

No estudo quantitativo, o pesquisador coleta e interpreta informações da realidade, valorizando o fenômeno em suas diferentes variáveis ${ }^{14}$.

Também foi realizado estudo comparativo entre os pacientes, divididos em dois grupos, os que receberam orientação pré-operatória de enfermagem e os que não a receberam.
O campo de pesquisa escolhido para a realização da coleta de dados abrangeu as enfermarias da clínica cirúrgica de um hospital universitário, no município do Rio de Janeiro, que presta atendimento para várias especialidades médicas e desenvolve ações de pesquisa e extensão. O estudo ocorreu no período de julho de 2012 a janeiro de 2013. A população estudada foi composta por pacientes submetidos à cirurgia geral no hospital estudado; a amostra foi constituída por 50 pacientes. Os critérios de inclusão foram: estar no período préoperatório para a realização de cirurgia geral, ter idade acima de 18 anos; estar lúcido, orientado. Foram excluídos desta pesquisa os pacientes da cirurgia pediátrica.

Como instrumento, foi utilizado um formulário com questões abertas para a caracterização do paciente, tipo de cirurgia e idade. A coleta de dados foi realizada com auxílio de um roteiro estruturado - o Inventário de Traço e Estado de Ansiedade (IDATE) de Spielberg, Gorsuch e Lushene - composto de duas escalas distintas elaboradas para medir os dois conceitos de ansiedade, ou seja, estado ansioso (IDATE-Estado) e traço ansioso (IDATE-Traço). Cada escala consiste em 20 afirmações para os quais os voluntários indicam a intensidade daquele momento (IDATE-Estado) ou a frequência com que ocorrem (IDATE- Traço) ${ }^{9,15,16}$.

A escala contém respostas às questões propostas do tipo Likert, que são não, um pouco, bastante e totalmente. Com base nelas, pontuações de 1 a 4 são atribuídas às respostas. Para ponto de corte na definição do grau de ansiedade utilizou-se os percentil 25 e 75 . O percentil 25 ou abaixo deste é considerado ansiedade baixa. Entre 25 e 75 considera-se ansiedade moderada e no percentil 75 ou acima deste considerase ansiedade elevada ${ }^{15}$. IDATE foi resultado de sucessivas verificações empíricas que estabeleceram dois tipos de ansiedade: ansiedade estado e ansiedade traço ${ }^{16}$.

Foi assegurado o anonimato dos participantes, assim como a total liberdade de resposta, podendo os mesmos interromperem a sua participação a qualquer momento, sem prejuízo para eles. A pesquisa foi realizada conforme as diretrizes do Conselho Nacional de Saúde do Ministério da Saúde ${ }^{17}$, tendo sido autorizada pelo Comitê de Ética em Pesquisa ao Hospital Universitário Pedro Ernesto do Estado do Rio de Janeiro (CEP/ HUPE/UERJ), com parecer no 3014/2011.

Os dados foram analisados e interpretados com o emprego da estatística descritiva simples, entendendo que os procedimentos estatísticos capacitam o pesquisador a reduzir, resumir, organizar, avaliar, interpretar e comunicar a informação numérica ${ }^{18}$.

A apresentação dos resultados se deu por meio de tabelas e figura, tendo sido aplicados cálculos de frequência absoluta e porcentual. As tabelas foram construídas, a partir do total de pacientes, para se obter também a média e o desvio padrão de traço e estado de ansiedade dos pacientes cirúrgicos hospitalares. 


\section{Resultados e Discussão}

Os sujeitos deste estudo foram constituídos por 50 pacientes, sendo que 15 (30\%) eram do sexo masculino e 35 (70\%) do sexo feminino. A idade variou entre 20 a 70 anos. Com relação à experiência cirúrgica pregressa, 41 (82\%) pacientes já haviam realizado cirurgias anteriormente e 9 (18\%), nunca. Ver a Tabela 1.

TABELA 1: Perfil dos pacientes submetidos à cirurgia geral. Rio de Janeiro, $2013(\mathrm{~N}=50)$.

\begin{tabular}{lcc}
\hline \multicolumn{1}{c}{ Características } & f & $\%$ \\
\hline Idade & 4 & 8,0 \\
$20-30$ & 7 & 14,0 \\
$31-40$ & 7 & 14,0 \\
$41-50$ & 23 & 46,0 \\
$51-60$ & 9 & 18,0 \\
$61-70$ & & \\
Sexo & 15 & 30,0 \\
Masculino & 35 & 70,0 \\
Feminino & & \\
Tipo de Cirurgia & 16 & 32,0 \\
Herniorrafia inguinal & 4 & 8,0 \\
Tireoidectomia & 20 & 40,0 \\
Colecistectomia & 2 & 4,0 \\
Paratireoidectomia & 4 & 8,0 \\
RTU de Bexiga & 4 & 8,0 \\
Hemorroidectomia & & 82,0 \\
Já realizaram Cirurgia? & 41 & 18,0 \\
Sim & 9 & \\
Não & & \\
\hline
\end{tabular}

A faixa etária mais frequente identificada no estudo foi de 51-60 anos - 23 (46\%), corrobando resultados de outros estudos, o que demonstra que esta população esta mais propensa a sofrer algum procedimento cirúrgico ${ }^{10}$.

Pode-se identificar que o procedimento cirúrgico mais frequente foi colecistectomia - 20 (40\%), seguido da herniorrafia inguinal - 16 (32\%). Com relação às cirurgias pregressas, a maior parte dos entrevistados já havia realizado algum ato cirúrgico, anteriormente.

A experiência prévia de um procedimento anestésico e cirúrgico e todas as suas implicações são vistas, portanto, como uma primeira exposição ao estressor, podendo influenciar nas estratégias de enfrentamento de outras cirurgias considerando seus níveis de complexidade $^{19}$.

Essas estratégias são utilizadas para diminuir as demandas internas e externas, contribuindo para minimizar os sentimentos de apreensão e solidão, fica distante da família e do trabalho, e por estar internado em um ambiente hospitalar e ser submetido a um procedimento cirúrgico que muitas vezes o paciente desconhece ${ }^{20}$.
Todos esses sentimentos contribuem para o aumento dos níveis de ansiedade. A determinação do traço e estado dos pacientes foi realizada com dois grupos, os pacientes que receberam orientações de enfermagem e os que não receberam orientações de enfermagem. Justamente com o intuito de conhecer os níveis de ansiedade intrínsecos (traço de ansiedade) e os níveis de ansiedade frente uma situação estressante com a cirurgia (estado de ansiedade).

A comparação ente traço e estado de ansiedade permitiu identificar os níveis de ansiedade, além de investigar também possíveis contribuições das orientações de enfermagem.

De acordo com os resultados obtidos, foi observado que os pacientes orientados apresentaram traço de ansiedade igual a média 36,2 e estado de ansiedade igual a média 35,6 mantendo próximo esses níveis (equilíbrio). Enquanto os pacientes não orientados apresentaram traço igual a média 36,6 e estado de ansiedade igual a média 44,6. Os pacientes não orientados apresentaram a diferença da média entre traço e estado aumentado em +8 em seus níveis de ansiedade, já os orientados tiveram a diferença da média de ansiedade reduzida em $+0,6$, como dispõe a Tabela 2. Por meio desses dados, pode-se perceber que os pacientes que receberam as orientações préoperatórias mostraram-se menos ansiosos quando comparados os índices de traço e estado de ansiedade dos pacientes não orientados.

TABELA 2: Distribuição da média por traço e estado dos pacientes submetidos à cirurgia geral hospitalar. Rio de Janeiro, 2013.

\begin{tabular}{lccc}
\hline \multicolumn{1}{c}{ Variáveis } & Média & $\begin{array}{c}\text { Desvio } \\
\text { Padrão }\end{array}$ & $\begin{array}{c}\text { Diferença } \\
\text { Média }\end{array}$ \\
\hline Orientados & & & \\
$\begin{array}{l}\text { Traço } \\
\text { Estado }\end{array}$ & 36,2 & 4,4 & \\
Traço/Estado & 35,6 & 2,3 & $+\mathbf{+ 0 , 6}$ \\
& & & \\
Não Orientados & & & \\
Traço & 36,6 & 2,8 & \\
Estado & 44,6 & 3,78 & $\mathbf{+ 8 , 0}$ \\
Traço/Estado & & & \\
\hline
\end{tabular}

Os sentimentos de ansiedade podem ser desenvolvidos no período pré-operatório, deixando o emocional do paciente abalado, contribuindo para a sua vulnerabilidade e dependência. Muitos pacientes apresentam alto nível de estresse, independente do grau de complexidade da cirurgia, o que pode ter uma relação com a desinformação dos procedimentos cirúrgicos, da anestesia e dos cuidados pré-operatórias ${ }^{21}$.

O nível de ansiedade dos pacientes durante o procedimento cirúrgico mostrou-se elevado quando 
não receberam as orientações de enfermagem, evidenciando a importância da atuação de enfermeiro nesse momento. Por sua vez, a ansiedade apresenta diferentes níveis: baixo, médio e alto de acordo com a Escala de Spielberg, Gorsuch e Lushene ${ }^{9,15,16}$. Dessa forma, passou-se a investigar o perfil dos níveis de ansiedade dos pacientes orientados e não orientados.

Observou-se que os níveis do traço e do estado de ansiedade dos pacientes orientados mantiveramse baixos, com percentuais respectivos no traço de ansiedade: baixo $(73,3 \%)$; médio $(26,7 \%)$; alto $(0 \%)$ e nos níveis de estado de ansiedade foram: baixo $(88,7 \%)$; médio $(13,3 \%)$; alto (0\%), evidenciando um aumento dos níveis baixo de estado de ansiedade dos pacientes orientados quando comparado com o traço de ansiedade. Enquanto os níveis de ansiedade-traço e estado dos pacientes não orientados apresentaram variações bastante significativas: traços de ansiedade alcançaram os resultados - baixo $(33,4 \%)$; médio $(66,6 \%)$; alto $(0 \%)$ - e estados de ansiedade - baixo $(6,6 \%)$; médio $(73,3 \%)$; alto $(20 \%)$-, um aumento dos níveis médios e altos de ansiedade.

Estes resultados reforçam ainda mais a relevância das orientações de enfermagem para os pacientes cirúrgicos, pois aqueles que recebem orientações mantêm níveis de ansiedade muito similares, enquanto os pacientes não orientados aumentam seus níveis de ansiedade, como demonstrada na figura 1. Os resultados mostram ainda que os pacientes não orientados sofrem uma diferença no perfil de ansiedade, quando comparados entre traço e estado de ansiedade. Os níveis médio e alto de ansiedade dos pacientes não orientados obtiveram aumento significativo.
As orientações de enfermagem auxiliam no esclarecimento quanto às dúvidas que a intervenção cirúrgica provoca, reduzem a duração da internação hospitalar, elevam a satisfação com o serviço prestado e minimizam as complicações pós-operatórias ${ }^{21}$.

Essas orientações podem ser realizadas pelos enfermeiros, das unidades de clinicas cirúrgicas e de centro cirúrgico, e devem ser realizadas individualmente, para o atendimento das necessidades de cada paciente; é preciso valorizar a visita de enfermagem pré-operatória com o paciente cirúrgico, estabelecendo um diálogo baseado na escuta terapêutica, proporcionando ao paciente um ambiente calmo e tranquilo para o esclarecimento de suas dúvidas ${ }^{21}$.

Entre as limitações do estudo, destacam-se a reduzida amostra e um único cenário que impedem a generalização dos resultados.

\section{Conclusão}

O estudo apresentou contribuições no intuito de facilitar a assistência de enfermagem perioperatória, colaborando para o saber da enfermagem e para a reflexão sobre mudanças no cuidar, uma vez que foi possível identificar o quanto a ansiedade pode influenciar o bem-estar do paciente.

O nível de ansiedade alto pode comprometer o sucesso da cirurgia, uma vez que gera sentimentos ruins, abalando o cliente. Foi identificado no estudo que os pacientes não orientados pela equipe de enfermagem aumentam de forma significativa seus níveis de ansiedade, revelando o emocional abalado, o que pode contribuir para possíveis complicações no pós-operatório.

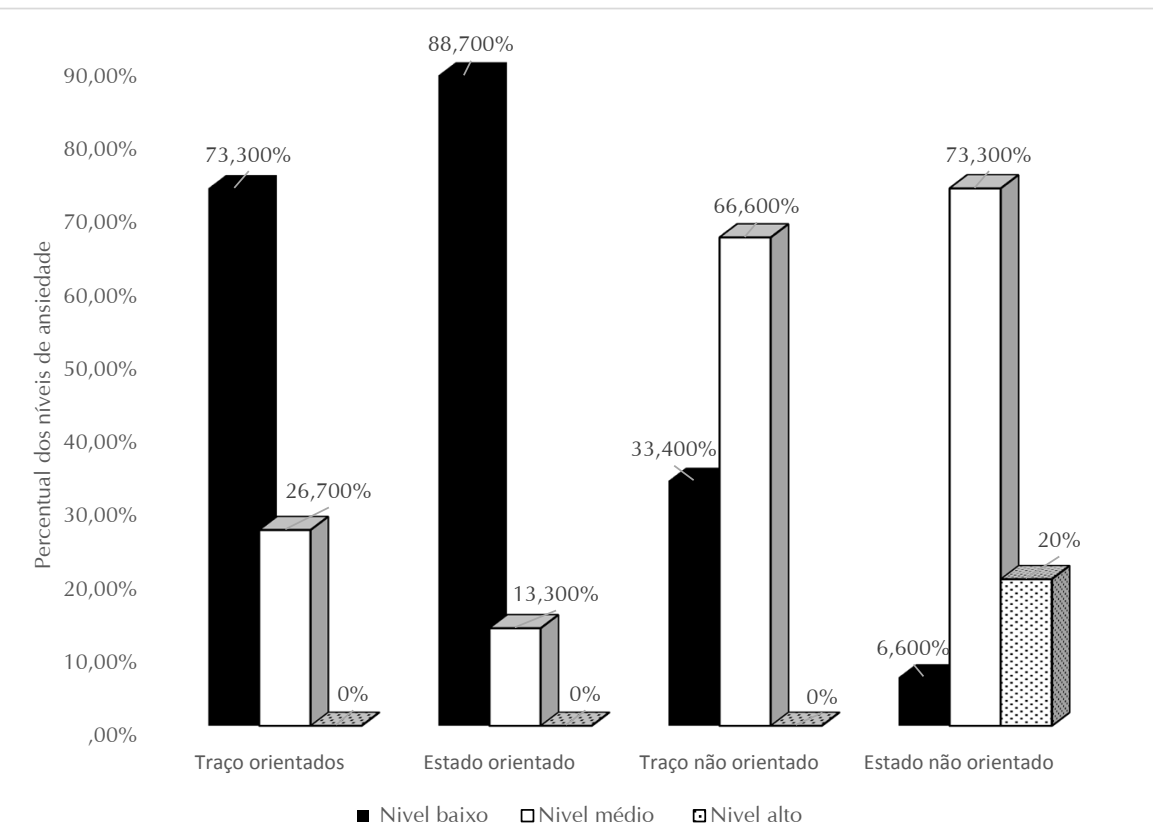

FIGURA 1: Percentual de pacientes submetidos à cirúrgica geral hospitalar e orientados e não orientados, de acordo com seu nível de ansiedade. Rio de Janeiro, 2013. 
Conclui-se que, para superar tais carências, devem ser criadas estratégias para fornecer orientações de enfermagem no pré-operatório, pois elas têm importância fundamental, tanto para o paciente cirúrgico como para toda a equipe que participa desse momento, com vistas ao bem-estar e à redução da ansiedade e dos riscos cirúrgicos.

\section{REFERÊNCIAS}

1.Figuera J, Viero VE. Vivências do paciente com relação ao procedimento cirúrgico: fantasias e sentimentos mais presentes. Rev SBPH. 2005; 8: 51-63.

2.Costa VASF, Silva SCFS, Lima VCP.O pré-operatório e a ansiedade do paciente: a aliança entre o enfermeiro e o psicólogo. Rev SBPH. 2010; 8: 77-85.

3.Grazziano ES, Bianchi ERF. Nível de ansiedade de clientes submetidos a ngiocoronariografia e de seus acompanhantes. Rev Latino-Am Enfermagem. 2004; 12: 168-74. 4.Sampaio CEP, Ribeiro DA. Perfil cirúrgico e fatores determinantes das suspensões de cirurgias gerais ambulatoriais: contribuições para assistência de enfermagem. Revista de Pesquisa: Cuidado é Fundamental, online 2012; 4: 293847. [citado em 9 jul 2014] Disponível em: http://www.seer. unirio.br/index.php/cuidadofundamental.

5.Barreto RASS, Araújo ACO, Suzuki K, Freitas VC. A necessidade de informação do cliente em pré-operatório de colecistectomia. Rev Min Enferm. 2010; 14: 369-75. 6.Ministério da Saúde (Br). Manual de Normas e Rotinas e Centro Cirúrgico Central de Material de Esterilização. Lavras (MG): Ministério da Saúde; 2007.

7.Almeida CEE, Nokibara MP, Ribeiro DA, Sampaio CEP. O cuidado de enfermagem associado à prescrição de enfermagem numa unidade de cirurgia cardíaca. Revista de Pesquisa: O Cuidado é Fundamental, online 2012; 3: 2510-20. [citado em 10 jul 2014] Disponível em: http:// www.seer.unirio.br/index.php/cuidadofundamental. 8.Ministério da Saúde (Br). Biblioteca Virtual em Saúde do Ministério da Saúde. Ansiedade. São Paulo: BVS 2011. [citado em 10 jul 2014] Disponível em: http:// bvsms.saude.gov.br/bvs/dicas../224_ansiedade.html.
9.Peniche ACG, Jouclas VMG, Chaves EC. A influência da ansiedade na resposta do paciente no período pósoperatório. Rev esc enferm USP. 1999; 33: 391-403.

10.Peniche ACG. Ansiedade e o paciente cirúrgico: análise das variáveis intervenientes [tese de doutorado]. São Paulo: Universidade de São Paulo; 2005.

11.Baggio MA, Teixeira A, Portella MR. Pré-operatório do paciente cirúrgico cardíaco: a orientação de enfermagem fazendo a diferença. Rev Gaúcha Enfem. 2001; 22: $122-39$.

12.Grittem L, Méier MJ, Gaievicz AP. Visita préoperatoria de enfermagem: percepções dos enfermeiros de um hospital de ensino. Cogitare Enferm. 2006; 11: 245-51. 13.Lobiondo-Wood G, Haber J. Pesquisa em enfermagem: métodos, avaliação críticas e utilização. 4a ed. Rio de Janeiro: Guanabara Koogan; 2001.

14.Polit DF, Beck CT, Hungler BP. Fundamentos de pesquisa em enfermagem, métodos, avaliação e pesquisa. 5a ed. Porto Alegre (RS): Artes Médicas; 2004.

15.Frias TFP, Costa CMA, Sampaio CEP. O impacto da visita pré-operatória de enfermagem no nível de ansiedade dos pacientes cirúrgicos. Rev Min Enferm. 2010; 13:76-83. 16.Fioravanti ACM. Propriedades psicométricas do inventário de ansiedade traço-estado IDATE [dissertação de mestrado] Rio de Janeiro: Pontifícia Universidade Católica do Rio de Janeiro; 2006.

17.Ministério da Saúde (Br). Conselho Nacional de Saúde. Normas de pesquisa envolvendo seres humanos-Resolução. no 466/2012. Bioética. Brasília (DF): Editora MS; 2012.

18.Polit DF, Beck CT, Hungler BP. Fundamentos de pesquisa em enfermagem: métodos, avaliação e utilização. 5aed. Porto Alegre (RS): Artmed; 2004.

19.Moraes LO, Peniche ACG. Ansiedade e mecanismos de coping utilizados por pacientes cirúrgicos ambulatoriais. Rev esc enferm USP. 2003; 37: 54-62.

20.Sampaio CEP, Costa TMN, Araújo D, Santoro DC. Mecanismos de enfrentamento desencadeados por pacientes em situação estressora: cirurgia ambulatorial. Rev enferm UERJ. 2013; 24: 515-20.

21.Chistóforo BEB, Carvalho DS. Cuidados de enfermagem aos pacientes cirúrgicos no período pré-operatório. Rev esc enferm USP. 2009; 43: 14-22. 